\title{
Dipole Moment Functions of HF and HI Molecules Using an Anharmonic Potential
}

\author{
Manfred H. Kluckner and B. Sesh Rao \\ Department of Physics, University of North Dakota
}

Z. Naturforsch. 38 a, 16-19 (1983); received August 10, 1982

Vibration-rotation wave functions for $\mathrm{HF}$ and $\mathrm{HI}$ are computed by solving the radial Schroedinger wave equation numerically using an anharmonic potential function with seven adjustable parameters. With these wave functions the matrix elements of $\left[\left(r-r_{\mathrm{e}}\right) / r_{\mathrm{e}}\right]^{n}$ are computed. These are then applied in a least squares fit to experimentally measured values of the electric dipole matrix elements to yield the dipole moment coefficients $M_{0}, M_{1}, \ldots, M_{5}$.

\section{Introduction}

The electric charge distribution in a heteronuclear diatomic molecule varies continuously because of simultaneous vibration and rotation. This causes a continuous variation of the electric dipole moment, which is conveniently expressed in a power series expansion about the equilibrium internuclear distance, $r_{\mathrm{e}}$, in the form

$$
M(r)=\sum_{n} M_{n} x^{n},
$$

where $x=\left(r-r_{\mathrm{e}}\right) / r_{\mathrm{e}}$, and $n=0,1,2,3, \ldots$. The quantities $M_{0}, M_{1}, M_{2}, \ldots$ are called the electric dipole moment coefficients with $M_{0}$ often referred to as the permanent dipole moment.

The value of the dipole moment function, $M(r)$, cannot be measured directly. However, it can be evaluated from the electric dipole matrix elements, $\mathfrak{M}_{v J}^{v^{\prime} J^{\prime}}$, via

$$
\mathfrak{M}_{v J}^{v^{\prime} J^{\prime}}=\sum_{n} M_{n} \int \Psi_{v^{*} J^{\prime}}^{*}(r) x^{n} \Psi_{v J}(r) r^{2} \mathrm{~d} r,
$$

where $\Psi_{v J}$ and $\Psi_{v^{\prime} J^{\prime}}$ are the radial wave functions of the lower and upper vibration-rotation states, and $v$ and $J$ are the vibrational and rotational quantum numbers, respectively. The dipole matrix elements can be calculated from experimentally obtained line intensities, $I_{v J}^{v^{\prime} J^{\prime}}$, using the relation

$$
I_{v J}^{v^{\prime} J^{\prime}}=\frac{8 \pi^{3} N_{v J} \omega_{v J}^{v^{\prime} J^{\prime}}}{3 h c(2 J+1)}|m|\left|\mathfrak{M}_{v J}^{v^{\prime} J^{\prime}}\right|^{2}
$$

Reprint requests to Dr. B. Sesh Rao, Department of Physics, University of North Dakota, Grand Forks, ND 58202, USA. where $N_{v J}=$ number of molecules in the state $(v, J)$, $\omega_{v J}^{v^{\prime} J^{\prime}}=$ frequency of the transition from state $(v, J)$ to state $\left(v^{\prime} J^{\prime}\right)$, expressed in $\mathrm{cm}^{-1}$, and $m=J+1$ for the R-branch and $-J$ for the P-branch.

From the electric dipole matrix elements, the dipole moment coefficients $M_{0}, M_{1}, \ldots, M_{5}$ were calculated using Eq. (2) in a least squares fit. The results are then compared with those obtained by Ogilvie et al. [1] and Niay et al. [2].

\section{Calculations}

The wave functions in (2) were calculated by solving the radial Schroedinger wave equation

$$
\frac{1}{r^{2}} \frac{\mathrm{d}}{\mathrm{d} r}\left(r^{2} \frac{\mathrm{d} \Psi}{\mathrm{d} r}\right)+\left\{-\frac{J(J+1)}{r^{2}}+\frac{2 \mu}{\hbar^{2}}[E-V(r)]\right\} \Psi=0,
$$

where $\mu$ is the reduced mass of the molecule and $E_{v J}$ is the energy of the vibration-rotation state. An anharmonic potential function was used for $V(r)$, in the form

$$
V(r)=h c a_{0} x^{2}\left(1+a_{1} x+a_{2} x^{2}+\ldots\right) .
$$

The first seven terms in this expansion were retained and therefore the first seven potential parameters $a_{0}, a_{1}, a_{2}, \ldots, a_{6}$ were determined. Calculations with eight and nine parameters were also attempted but did not lead to any significantly improved results within a reasonable amount of computer time.

The equilibrium internuclear distance, $r_{\mathrm{e}}$, was calculated from the rotational constant

$$
B_{\mathrm{e}}=\frac{h}{8 \pi^{2} c \mu r_{\mathrm{e}}^{2}} \text {. }
$$


The numerical value of $B_{\mathrm{e}}$ as well as other molecular constants were taken from papers by Webb and Rao [3] for HF and by Bernage et al. [4] for HI.

Initial starting values for $E_{v J}$ were calculated using Dunham's [5] expression

$$
E_{v J}=\sum_{l, j} Y_{l j}\left(v+\frac{1}{2}\right)^{l} J^{j}(J+1)^{j}
$$

where the $Y_{l j}$ 's are the Dunham coefficients, which are very nearly equal to the molecular constants.

Approximate starting values for the potential parameters, $a_{i}$, were calculated using formulae provided by Guenther [6]. These formulae express the $a_{i}$ 's in terms of the $Y_{l j}$ 's.

Then the procedure described below was used to solve for the wave functions and eigenvalues in Equation (4).

(a) Following Loucks [7], a fourth-order RungeKutta method in combination with Milne method was used to solve Equation (4) from a point near the origin ( $r_{0}=10^{-8} a_{\mathrm{B}}, a_{\mathrm{B}}=$ Bohr radius) outward, and also inward from a suitably chosen value of $r\left(r_{\mathrm{inf}}=\right.$ $6.0 a_{\mathrm{B}}$ ). The starting values mentioned above were used for $E_{v J}$ and $a_{i}$. At $r_{\mathrm{e}}$, the logarithmic derivatives for the two solutions, one approaching from the origin and the other from "infinity" $\left(r_{\text {inf }}\right)$, were evaluated and compared.

(b) Step (a) was repeated with different successively adjusted values of $E_{v J}$ until the two logarithmic derivatives were equal. The value of $E_{v J}$ satisfying this condition was accepted as an eigenvalue of the radial wave equation. In this fashion a number of different energy levels were obtained from which various transition frequencies were computed.

(c) The calculated transition frequencies were compared to the measured values, which were taken from papers by Hurlock et al. [8], Haeusler et al. [9], and Bernage et al. [4] in the case of HI, and publications by Webb and Rao [3] and Fishburne and Rao [10] for HF. The standard deviation between calculated and measured frequencies was computed to serve as a measure of agreement between these two sets of data.

(d) The procedure described in steps (a), (b) and (c) was repeated for successively adjusted potential parameters over and over again until there was satisfactory agreement between the experimental and the calculated values of the frequencies. In mathematical terms, the problem was to minimize the standard deviation of step (c). Because of the sizeable number of potential parameters involved in this work, a numerical routine from the International Mathematical and Statistical Libraries (IMSL) was added to the computer program to carry out this tedious task. This routine, called ZXMIN, is based on a Quasi-Newton algorithm developed by Fletcher [11]. An advantage of this particular routine is that it does not require a usersupplied gradient.

All the relevant eigenvalues and wave functions for $\mathrm{HF}$ and $\mathrm{HI}$ were calculated using the final sets of parameters obtained in (d). Proper subroutines were added to the computer program to normalize the wave functions. The orthogonality condition was tested by calculating various integrals of the form $\left\langle v J \mid v^{\prime} J^{\prime}\right\rangle$. These and the integrals in Eq. (2) were evaluated numerically using Simpson's rule.

The electric dipole moment coefficients, $M_{n}$, were now extracted from a least squares fit of the right hand side side of Eq. (2) to the experimentally obtained values of the dipole matrix elements $\mathfrak{M}_{v J}^{v^{\prime} J^{\prime}}$ on the left side, in the sense

$$
\sum_{\substack{v^{\prime} J^{\prime} \\ v J}}\left[\mathfrak{M}_{v J}^{v^{\prime} J^{\prime}}-\sum_{n} M_{n} \int \Psi_{v^{\prime} J^{\prime}}^{*} x^{n} \Psi_{v J} r^{2} \mathrm{~d} r\right]^{2}=\min .
$$

It was found that $M_{0}, M_{1}, \ldots, M_{k}$ can be obtained if the least squares fit involves a matrix element in the vibrational ground state, which is available from Stark effect measurements, and further matrix elements from $k$ number of bands.

In the case of $\mathrm{HF}$, the matrix element $\mathfrak{M}_{01}^{01}$ was measured by Muenter and Klemperer [12]. The other matrix elements were calculated using Eq. (3) from measured line intensities. The line intensities of the $(1-0)$ band were measured by Lovell and Herget [13], those of the $(2-0)$ band by Meredith [14], and those of the $(3-0)$ band by Spellicy et al. [15]. For the $(4-0)$ and $(5-0)$ bands only the rotationless matrix elements, $\mathfrak{M}_{00}^{40}$ and $\mathfrak{M}_{00}^{50}$, obtained by Rimpel [16], were utilized. A total of $51 \mathrm{HF}$ vibration-rotation matrix elements were used.

For HI, $\mathfrak{M}_{00}^{01}$ had been obtained by van Dijk and Dymanus [17]. The line intensities for the $(1-0)$ band were measured by Ameer and Benesch [18], the ones in the $(2-0)$ and $(3-0)$ bands by Meyer et al. [19], and those in the $(4-0)$ and $(5-0)$ bands by Niay et al. [2]. A total of $101 \mathrm{HI}$ matrix elements were available. 
The question arises then, why only 6 coefficients were extracted from a least squares fit to such a sizeable number of vibration-rotation matrix elements. The differences between matrix elements of the same band are small compared to the differences between the elements of different bands. The integrals in Eq. (2) show a similar behavior. So the $51(\mathrm{HF})$ or $101(\mathrm{HI})$ equations involved in the fit essentially break down into 6 sets of equations. Attempts were made to solve for more than 6 coefficients, but they did not lead to meaningful results because of ill-conditioning problems.

In the past, Ogilvie et al. [1] and Niay et al. [2] have determined the coefficients $M_{0}, M_{1}, \ldots, M_{5}$ using six equations involving six rotationless dipole matrix elements and analytical expressions for the integrals $\left\langle v^{\prime} \mathbf{0}\left|x^{n}\right| 00\right\rangle$. For comparison, a similar calculation was carried out here, but using numerically obtained integrals.

In the least squares fit one last problem concerned the signs of the dipole matrix elements. Since the matrix elements were obtained from the line intensities, which actually depend on the squares of the matrix elements, there is an ambiguity in the signs of the matrix elements. This was handled by assuming a convention similar to that used by Cashion [20]. In the case of HF the signs of the matrix elements were chosen as +-+-+ for the $(1-0)$, $(2-0),(3-0),(4-0)$ and $(5-0)$ bands, respectively, while for HI they were taken as -+- +-.

The least squares fit was carried out for various numbers of coefficients and each time the standard deviation between the calculated and the experimental values of the matrix elements was computed. The fit involving 6 coefficients led to the best results.

Finally, to assess the accuracy of the dipole moment coefficients obtained in this work, an error estimate was carried out. The effect of the errors of the measured values of the dipole matrix elements on the final set of dipole moment coefficients was investigated and standard errors were calculated. This calculation was carried out along the lines of Pugh and Winslow [21].

\section{Results and Discussion}

The potential parameters $a_{0}, a_{1}, \ldots, a_{6}$, arrived at in this work are listed in Table 1. For comparison, the parameters used by Ogilvie et al. [1] are also
Table 1. Potential parameters for HF and $\mathrm{HI}\left(a_{0}\right.$ in $\mathrm{cm}^{-1}$; $a_{1}, a_{2}, \ldots$ dimensionless).

\begin{tabular}{lrcrrr}
\hline \multicolumn{2}{l}{$\mathrm{HF}$} & \multicolumn{3}{l}{$\mathrm{HI}$} \\
\cline { 2 - 3 } \cline { 5 - 6 } $\begin{array}{l}\text { Our param- } \\
\text { eters }\end{array}$ & $\begin{array}{l}\text { Ogilvie } \\
\text { et al. [1] }\end{array}$ & & $\begin{array}{l}\text { Our param- } \\
\text { eters }\end{array}$ & $\begin{array}{l}\text { Ogilvie } \\
\text { et al. [1] }\end{array}$ \\
\hline$a_{0}$ & 204138.0669 & 204348.50 & 204751.7326 & 204675.50 \\
$a_{1}$ & -2.250014007 & -2.2538 & -2.533502310 & -2.54625 \\
$a_{2}$ & 3.499498195 & 3.4882 & & 3.901170077 & 4.05336 \\
$a_{3}$ & -4.561790849 & -4.4986 & & -5.205232826 & -5.4421 \\
$a_{4}$ & 4.605981387 & 4.704 & & 9.878919543 & 6.6407 \\
$a_{5}$ & -3.127738565 & -2.91 & & -21.702937036 & -6.9146 \\
$a_{6}$ & 1.425362094 & -1.76 & & 21.719851192 & 0.38 \\
\hline
\end{tabular}

included. Only in the case of $a_{5}$ and $a_{6}$ for $\mathrm{HI}$, and $a_{6}$ for HF, there is a sizeable difference between the two sets of parameters. Calculations with eight and nine parameters were also attempted but did not lead to any significantly improved results within a reasonable amount of computer time. With these final seven parameters, a large number of energy eigenvalues and transition frequencies of various vibration-rotation bands were calculated for both molecules. It was found that the standard deviation between these calculated and the measured frequencies was roughly of the same order of magnitude as the standard deviation in the experimental data.

The dipole moment coefficients obtained from the least squares fit are listed in Tables 2 and 3 along with those found by solving the six equations

Table 2. Values of the dipole moment coefficients of HF in Debye (numbers in parentheses are standard errors).

\begin{tabular}{|c|c|c|c|}
\hline & \multicolumn{2}{|l|}{ Our results } & \multirow{2}{*}{$\begin{array}{l}\text { Ogilvie et al. [1] } \\
\text { (six equations) }\end{array}$} \\
\hline & $\begin{array}{l}\text { From least } \\
\text { squares fit }\end{array}$ & $\begin{array}{l}\text { From system } \\
\text { of six equa- } \\
\text { tions }\end{array}$ & \\
\hline$M_{0}$ & $\begin{array}{c}1.80306 \\
( \pm 0.00006)\end{array}$ & 1.80298 & 1.80306 \\
\hline$M_{1}$ & $\begin{array}{c}1.39079 \\
( \pm 0.00246)\end{array}$ & 1.38921 & 1.39366 \\
\hline$M_{2}$ & $\begin{array}{c}-0.06916 \\
( \pm 0.00193)\end{array}$ & -0.06388 & -0.0583 \\
\hline$M_{3}$ & $\begin{array}{c}-0.8668 \\
( \pm 0.0076)\end{array}$ & -0.9027 & -0.8861 \\
\hline$M_{4}$ & $\begin{array}{c}-0.5295 \\
( \pm 0.0089)\end{array}$ & -0.5722 & -0.599 \\
\hline$M_{5}$ & $\begin{array}{c}-1.077 \\
( \pm 0.063)\end{array}$ & -0.952 & -0.931 \\
\hline
\end{tabular}


Table 3. Values of the dipole moment coefficients of HI in Debye (numbers in parentheses are standard errors.)

\begin{tabular}{|c|c|c|c|c|}
\hline & \multicolumn{2}{|l|}{ Our results } & \multirow{2}{*}{$\begin{array}{l}\text { Ogilvie } \\
\text { et al. (six } \\
\text { equations) } \\
{[1]}\end{array}$} & \multirow{2}{*}{$\begin{array}{l}\text { Niay et al. } \\
\text { (six equa- } \\
\text { tions) [1] }\end{array}$} \\
\hline & $\begin{array}{l}\text { From least } \\
\text { squares fit }\end{array}$ & $\begin{array}{l}\text { From } \\
\text { system of } \\
\text { six equa- } \\
\text { tions }\end{array}$ & & \\
\hline$M_{0}$ & $\begin{array}{c}0.44721 \\
( \pm 0.00001)\end{array}$ & 0.44727 & 0.44722 & $\begin{array}{c}0.44728 \\
( \pm 0.00005)\end{array}$ \\
\hline$M_{1}$ & $\begin{array}{c}-0.07252 \\
( \pm 0.00022)\end{array}$ & -0.07406 & -0.07404 & $\begin{array}{r}-0.073389 \\
( \pm 0.00032)\end{array}$ \\
\hline$M_{2}$ & $\begin{array}{c}0.51921 \\
( \pm 0.00027)\end{array}$ & 0.50608 & 0.5057 & $\begin{array}{r}0.5028 \\
( \pm 0.0036)\end{array}$ \\
\hline$M_{3}$ & $\begin{array}{c}-2.0691 \\
( \pm 0.0002)\end{array}$ & -2.0111 & -1.9794 & $\begin{array}{c}-1.9898 \\
( \pm 0.018)\end{array}$ \\
\hline$M_{4}$ & $\begin{array}{r}0.1159 \\
( \pm 0.0002)\end{array}$ & -0.0376 & -0.053 & $\begin{array}{l}-0.1379 \\
( \pm 0.07)\end{array}$ \\
\hline$M_{5}$ & $\begin{array}{c}2.4898 \\
( \pm 0.0002)\end{array}$ & 0.5833 & -0.0015 & $\begin{aligned} & 1.1467 \\
&( \pm 0.34)\end{aligned}$ \\
\hline
\end{tabular}

involving rotationless matrix elements. For comparison, the results of Ogilvie et al. [1] and Niay et al. [2] are also included in the tables. The numbers in parentheses are the standard errors. In the case of

[1] J. F. Ogilvie, W. R. Rodwell, and R. H. Tipping, J. Chem. Phys. 73, 5221 (1980).

[2] P. Niay, P. Bernage, C. Coquant, and R. Houdart, Can. J. Phys. 56, 727 (1978).

[3] D. U. Webb and K. N. Rao, J. Mol. Spectrosc. 28, 121 (1968).

[4] P. Bernage, P. Niay, and R. Houdart, C. R. Acad. Sci. Paris 278, 235 (1974).

[5] J. L. Dunham, Phys. Rev. 41, 721 (1932).

[6] A. H. Guenther (private communication).

[7] T. L. Loucks, Augmented Plane Wave Method. W. A. Benjamin, Inc., New York 1967.

[8] S. C. Hurlock, R. M. Alexander, K. N. Rao, and Sr. N. Dreska, J. Mol. Spectrosc. 37, 373 (1971).

[9] C. Haeusler, C. Meyer, and P. Barchewitz, J. Phys. Paris 25, 961 (1964).

[10] E. S. Fishburne and K. N. Rao, J. Mol. Spectrosc. 19, 290 (1966).

[11] E. S. Fletcher, Fortran Subroutines for Minimization by Quasi-Newton Methods, Report R7125 AERE, Harwell, England 1972.
HF the three sets of coefficients listed in Table 2 are in good agreement, especially $M_{0}$ and $M_{1}$. The coefficients obtained by Ogilvie et al. [1] are however somewhat closer to the ones obtained from the six equations in the present work than to the least squares fit results. This effect is more pronounced for the coefficients listed for HI, especially for $M_{4}$ and $M_{5}$. Vibration-rotation interaction is the most likely reason for this behavior. These findings suggest that the dipole moment coefficients $M_{0}, M_{1}$, $M_{2}$ and $M_{3}$ can be determined very reliably from a system of 6 equations utilizing a ground state matrix element and the quantities $\mathfrak{M}_{00}^{10}, \mathfrak{M}_{00}^{20}, \ldots, \mathfrak{M}_{00}^{50}$, but for the higher order coefficients $M_{4}, M_{5}$ etc., vibration-rotation interaction becomes increasingly significant so that a least squares fit making use of a sizeable number of vibration-rotation matrix elements might lead to better results in these cases.

In conclusion, the dipole moment coefficients obtained using the 51 (for HF) and 101 (for HI) dipole matrix elements are chosen as the final sets since they were extracted from a very large number of vibration-rotation matrix elements instead of just six rotationless ones.

[12] J. S. Muenter and W. Klemperer, J. Chem. Phys. 52, 6033 (1970).

[13] R. J. Lovell and W. F. Herget, J. Opt. Soc. Amer. 52, 1374 (1962)

[14] R. E. Meredith, J. Quant. Spectrosc. Radiat. Transfer. 12, 485 (1972).

[15] R. L. Spellicy, R. E. Meredith, and F. G. Smith, J. Chem. Phys. 57, 5119 (1972).

[16] G. Rimpel, Z. Naturforsch. 29 a, 588 (1974).

[17] F. A. van Dijk and A. Dymanus, Chem. Phys. Letters 5, 387 (1970).

[18] G. Ameer and W. Benesch, J. Chem. Phys. 37, 2699 (1962).

[19] C. Meyer, C. Haeusler, and P. Barchewitz, J. Phys. Paris 26, 305 (1965).

[20] J. K. Cashion, J. Mol. Spectrosc. 10, 182 (1963).

[21] E. M. Pugh and G. H. Winslow, The Analysis of Physical Measurements, Addison-Wesley, Inc., Reading (Ma.) 1966. 